\title{
Message from the Chairs
}

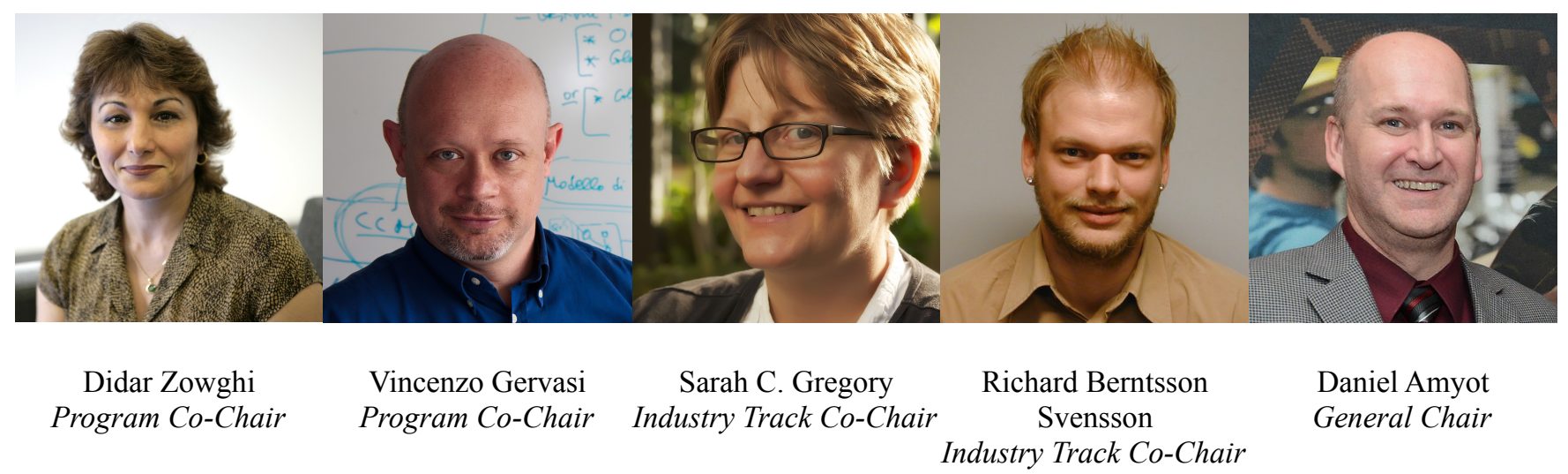

It is with great pleasure that we welcome you to the 23rd IEEE International Requirements Engineering Conference, RE'15, in the beautiful multicultural city of Ottawa, Canada.

For almost a quarter of a century now, this Conference has hosted researchers, practitioners, educators and students interested in Requirements Engineering, presenting latest results, technical innovations, reports from field work, emerging challenges and opportunities. In so doing, RE has often opened the way for tool vendors, companies, teaching institutions, and professional bodies looking to anticipate the needs of the booming information society at large.

The particular focus of RE'15 is in line with the special role that Requirements Engineering can play in identifying the needs of large population segments, and in effectively processing their input, expressed or implied, and to deliver better products and achieve greater satisfaction to a large number of users. This special focus is distilled in the RE'15 motto of "Requirements for the masses, requirements from the masses." - a theme which several accepted papers have addressed.

The conference offers a rich technical program, including a number of selected papers from (and for) both academic and industrial perspectives. Presentations of these papers, grouped by subject, span the entire 3-day duration of the conference. In addition, this year we have introduced a new track named "RE: Next!" that offers shorter previews of ongoing work. This track is an excellent opportunity to hear what people around the world are currently working on, to establish early collaborations, to stimulate ideas and to share experiences while the chances of influencing future development are high, or even to get the first peek at the next big breakthrough!

A number of complementary events enrich and complete our program: tutorials, posters, a doctoral symposium, tools \& demo highlights, discussion panels, and reports "from the trenches" about the current state of practice. Ten workshops, on Monday and Tuesday, provide excellent opportunities for more focused presentations and discussions. Some of the workshops have long been associated with RE, and we are glad of continuing such a fruitful tradition; others are being held for the first time. To the latter goes our special welcome to the RE family!

We are honored to have three excellent keynote speakers this year; each of them will offer a different take on the RE'15 theme. On Wednesday, Aydin Y. Mirzaee (General Manager, FluidReview, SurveyMonkey) will tell the story of how FluidReview and then SurveyMonkey have managed their requirements and product roadmap, in a context where the company has tens of millions of users and 
stakeholders. On Thursday, Karen Holtzblatt (Founder \& CEO, InContext) will talk about Contextual Design, a set of design principles based on a deep understanding of users' contexts. On Friday, Michael Jackson (Independent Consultant and Visiting Research Professor, The Open University) will share his reflections about how RE can be rethought in terms of meeting the needs and desires of large numbers of stakeholders in cyberphysical systems.

Overall, RE'15 attracted 228 abstract submissions (129 in the Research track, 27 in the Industry track, 72 in the RE: Next! track), which were followed by 171 full papers (97 Research, 23 Industry, 51 Next). Papers in the Research track were independently reviewed by three members of the Program Committee, with reviews followed by a period of online discussion open to all PC members. All papers that had at least one positive review at the end of the discussion period were examined at the plenary Research PC Meeting that was held in Pisa, Italy, on the 14th and 15th of May. 19 papers from this category were finally accepted for presentation at the Conference (an acceptance rate of 19.6\%). Papers in the Industry track were also reviewed by three members of the Program Committee and then discussed online, and acceptance was based on their reviews (with additional reviews solicited in a small number of cases); 9 papers were finally accepted for presentation (acceptance rate of 39.1\%). Finally, RE: Next! communications were reviewed by two members of the Program Committee. All papers with two positive recommendations were accepted; those with one positive and one negative recommendation received a further review. In the end, 15 communications were included in the program (acceptance rate of 29.4\%).

The outcome of the selection process consisted of 43 papers grouped into 16 thematic sessions, which together provide a broad and deep view on current advancements in Requirements Engineering.

We received submissions from 500 authors, from 28 different countries: 40\% from Europe, 23\% from the Americas, 8\% from Asia, 4\% from Oceania, 1\% from Africa; in almost 24\% of the cases we could not identify a specific country. As for accepted papers, the US provided the largest individual contribution, with 11 papers, followed by Germany and Sweden with 8 and 6 papers respectively. Overall, 16 different countries are represented in the main conference program, with many other countries represented in the various workshops and other events.

We would like to express our appreciation to the great effort exerted by the members of the Program Committee (PC) in dedicating their time to provide valuable reviews. This year, the membership of the $\mathrm{PC}$ contained many of the most experienced RE researchers and practitioners as well as many enthusiastic younger researchers who have joined the RE PC for the first time. To all 77 members of the PC (47 for the Research track, 19 for the Industry track, 11 for the RE: Next! track) we give our warmest gratitude for their invaluable work.

We would also like to extend a special thanks to the members of the Organizing Committee and of the many sub-committees they created: without their help and expertise, there would be no RE'15. We thank in advance the 21 student volunteers selected for helping with the smooth running of the conference, as well as many other volunteers who are generously giving their time to ensure the success of RE'15. Last but not least, we also thank our corporate and academic sponsors and donors for their generous support.

Finally, we sincerely hope that RE' 15 attendees, and especially those participating to RE for the first time, will benefit from an enriching experience and will enjoy the conference and Ottawa to the fullest extent!

Didar Zowghi and Vincenzo Gervasi, Program Co-Chairs Sarah C. Gregory and Richard Berntsson Svensson, Industry Track Co-Chairs Daniel Amyot, General Chair 